\title{
Eficacia-efectividad del programa de reducción del estrés basado en la conciencia plena (MBSR): actualización.
}

\author{
Efficacy-effectiveness of mindfulness based stress reduction (MBSR) programme: an update.
}

\author{
José Manuel Bertolín Guillén a . \\ a Servicio de Psiquiatría y Salud Mental, Hospital Arnau de Vilanova, Generalitat Valenciana. Valencia, \\ España.
}

Correspondencia: José Manuel Bertolín Guillén (jmbertolin@comv.es)

Recibido: 28/04/2014; aceptado: 03/11/2014

\begin{abstract}
RESUMEN: El programa de reducción del estrés basado en la conciencia plena o mindfulness based stress reduction (MBSR) es una intervención instructiva o psicoeducativa, estructurada, multimodal e inespecífica, diseñada para mejorar la conciencia plena de los participantes con focalización en el presente. Hay pruebas de su efectividad en el dolor crónico (incluyendo la fibromialgia), depresiones recurrentes o con síntomas residuales, el estrés en general y en diversos trastornos de ansiedad, así como en ciertas condiciones médicas diversas como el cáncer, epilepsia resistente, psoriasis y enfermos con VIH, entre otras. El cuerpo probatorio que se ha acumulado hasta la fecha es muy prometedor y fuertemente indiciario de los beneficios del MBSR en el corto y medio plazo, pero se necesitan más estudios adecuados para confirmar su bondad por tiempos prolongados, y estudios potentes de buena calidad metodológica que confirmen su eficacia y efectividad.
\end{abstract}

PALABRAS CLAVE: mindfulness; conciencia plena; reducción del estrés basado en la conciencia plena; MBSR; meditacion; evaluación de programa.
ABSTRACT: The mindfulness based stress reduction (MBSR) programme is a structured, multimodal and unespecific instructive or psychoeducative intervention. It has been designed to improve the participants' mindfulness focused on the present time. Its effectiveness has been proved in chronic pain (including fibromyalgia), recurrent depression or with residual symptoms, stress in general and in diverse anxiety disorders, as well as in certain medical conditions such as cancer, resistant epilepsy, psoriasis and HIV, among others. The evidential body that has been accumulated to date is highly promising strongly indicative of the benefits of MBSR in the short and medium term. However, more appropriate studies are needed in order to confirm the benefits of MBSR in the long term. Powerful studies with good methodological quality are also needed in order to confirm the efficacy and effctiveness of MBSR.

KEY WORDS: mindfulness; mindfulness training; mindfulness-based stress reduction (MBSR); meditation; program evaluation.

\section{Introducción}

El programa de reducción del estrés basado en la conciencia plena o mindfulness based stress reduction (MBSR) está fundamentado en la meditación vipassa$n a$ y fue la primera de las intervenciones terapéuticas que se desarrolló basada en la conciencia plena. Se trata de un programa psicoeducativo, no de un verdadero tratamiento psicoterapéutico per se y representa un enfoque genérico o inespecífico de intervención. El programa se inició hace más de treinta años en EEUU para aliviar el dolor y el estrés relacionado con ciertas enfermedades crónicas. Su fundador fue Jon Kabat-Zinn, quien encuadra su programa dentro de la denominada medicina 
del comportamiento o medicina conductual (1). El programa está basado en la comunidad y parece relativamente costo-efectivo. Consta de ocho sesiones semanales de unas dos horas o dos horas y media de duración, además de trabajo en casa, pero se conjetura si una forma abreviada del mismo sería igualmente eficaz para ciertos pacientes (2). El componente de conciencia plena o mindfulness del programa, que es completamente secular, consta de tres prácticas diferentes: meditación sentada o caminando, exploración del cuerpo o body scan y hatha yoga. Además se practica la meditación informal, que consiste en aplicar la conciencia plena a todas las actividades de la vida cotidiana.

La literatura especializada acerca del MBSR es de cantidad creciente pero de calidad irregular, de modo que sigue siendo necesario examinar la mejor información disponible sobre el tópico de este trabajo para disminuir la incertidumbre y contradicciones relacionadas. El objetivo del presente estudio es analizar la literatura especializada publicada en artículos de revista de la mejor calidad disponible para interpretar críticamente la eficacia-efectividad del programa en el momento actual.

\section{Material y método}

El punto de partida es una búsqueda en PubMed hasta julio de 2013 del conjunto de artículos obtenido con el descriptor mindfulness based stress reduction mbsr $(\mathrm{n}=$ 186). Se han añadido otros metaanálisis y revisiones sistemáticas que no aparecían en la búsqueda citada. Desglosados por tipos, los artículos hallados quedan distribuidos así: metaanálisis $(\mathrm{n}=8)$; revisiones sistemáticas $(\mathrm{n}=13)$; ensayos clínicos controlados y aleatorizados $(\mathrm{n}=52)$; ensayos clínicos $(\mathrm{n}=15)$. Por regla general se han eliminado los artículos que no se incluían en las categorías citadas. En el cómputo se han depurado los duplicados: cuando un artículo está incluido en un tipo superior (es superior si apare por delante en el listado anterior) se elimina del tipo inferior en que aparece también. Los ensayos clínicos controlados aleatorizados incluidos en este trabajo se señalan con un asterisco [*] al final de cada referencia en el apartado de bibliografía; las revisiones sistemáticas se señalan con dos y los metaanálisis con tres. Los ensayos clínicos no controlados, por último, se señalan con [+].

El estudio es una revisión narrativa, no sistemática, de tipo cualitativo, con componentes integradores, agregativos e interpretativos. Para la exposición de los resultados y su discusión se ha tenido en cuenta el total de artículos hallados, añadiendo otros trabajos $(\mathrm{n}=48)$ de menor calidad metodológica ("literatura gris") pero que aportan datos complementarios o avances de interés, no incluidos en los anteriores, con lo que el total de trabajos incluidos en esta revisión es finalmente de 
$\mathrm{n}=136$. Las revisiones sistemáticas y metaanálisis consultados permiten mejorar la exhaustividad del tópico investigado y controlar el sesgo de publicación. Los ensayos clínicos proporcionan buenas pruebas de intervención, de hipótesis y de evaluación, y representan el núcleo fundamental de esta revisión. La literatura gris ha permitido ampliar aún más, subjetivamente, el rango de búsqueda y contribuir a mejorar el sesgo del idioma.

\section{Resultados}

Se recogen estudios de calidad científica variable que han mostrado eficacia o efectividad del MBSR en trastornos y condiciones médicas muy diversas, incluyendo la cronicidad (3-4), y en mejorar la salud mental en general (5) y de los adolescentes en particular (6). Dos metaanálisis han estudiado los efectos de MBSR en la salud mental (7-8). El de Grossman et al. (8) se basó en 20 trabajos controlados y no controlados sobre los efectos de MBSR en la salud física y mental de muestras médicas y no médicas. Se halló un tamaño del efecto de 0,54 , pero es posible que estuviera sobrestimado al haberse incluido estudios cuasi-experimentales.

\section{Dolor crónico}

Ha sido bien establecida (9) la eficacia de las intervenciones psicoterapéuticas en general y de los tratamientos cognitivo-conductuales y de autorregulación en concreto, tanto en el dolor crónico lumbar no canceroso como en la discapacidad y alteraciones anímicas consiguientes. Con respecto al MBSR hay ciertas pruebas de que es también eficaz en la tolerancia al dolor y específicamente en el dolor crónico (10-15), incluyendo la fibromialgia (16-19). En relación con esta última se ha hallado (20) mejora de los síntomas depresivos asociados a la fibromialgia, pero algunos autores (21) no han probado la eficacia del MBSR en ella, aunque sí ciertos beneficios inespecíficos. Morone et al. (22) han mostrado resultados prometedores del MBSR en una población de adultos mayores de 65 años de edad con dolor crónico lumbar. Wong et al. (23) hallaron mejora en la intensidad del dolor y angustia relacionada, pero que no fue superior a una intervención interdisciplinar sobre el dolor. En la revisión sistemática de Cramer et al. (24) sobre un total acumulado de 117 pacientes con dolor lumbar crónico tampoco hubo pruebas concluyentes de la efectividad del MBSR en la mejora de la intensidad del dolor o de la discapacidad asociada, y sí pruebas limitadas de que puede mejorar la aceptación del dolor. En el metaanálisis de Veehof et al. (25) el MBSR no fue superior al tratamiento cognitivo-conductual, pero sí podía ser una alternativa eficaz.

Baer (7) incluyó en su revisión estudios controlados y no controlados sobre poblaciones con diversas enfermedades. En general, los pacientes con dolor crónico pre- 
sentaron mejoras estadísticamente significativas en las calificaciones de dolor, otros síntomas médicos y síntomas psíquicos generales. Muchos de esos cambios se mantuvieron en las evaluaciones de seguimiento. La mayoría de estas comparaciones utilizó diseños pre-post sin grupo control. Se ha sugerido en un estudio piloto en que se comparaba el MBSR con el masaje en pacientes con dolor crónico, que el primero puede ser más efectivo y de efectos más duraderos en la mejoría del humor mientras el masaje puede serlo más en reducir el dolor (26). El MBSR puede ser también una intervención clínicamente útil en el "síndrome de cirugía fallida de espalda" (failed back surgery syndrome o FBSS), que se define como dolor de espalda o pierna que persiste o reaparece después de uno o más procedimientos quirúrgicos en la columna lumbosacra (27).

2. Estrés y ansiedad

En un ensayo clínico controlado aleatorizado de pequeño tamaño muestral (28) se analizó si los individuos con grados más altos de conciencia plena pretratamiento se beneficiarían más de la intervención con el programa. Los resultados mostraron que los participantes con mayor grado de conciencia plena pretratamiento presentaron también mayor incremento en la atención, bienestar subjetivo, empatía y esperanza, y presentaron asimismo más disminución del estrés percibido hasta un año después de la intervención. La utilidad del programa sobre el desarrollo de la empatía ya se había sugerido antes (29-30). En escolares mejora la sensación subjetiva de bienestar (31). También en escolares (32) y en estudiantes de enfermería (33-34) parece una intervención eficaz para disminuir el estrés y la ansiedad. Por último, el programa ha sido eficaz asimismo para mejorar el grado de autocompasión en un estudio realizado con psicoterapeutas en formación (35).

En el metanálisis de Chiesa y Serretti (36) se revisaron las evidencias acerca de que el MBSR fuera eficaz para reducir el estrés en poblaciones no clínicas. Sin embargo, las importantes limitaciones de los estudios incluidos en esta revisión, así como la escasez de pruebas sobre los posibles efectos específicos del programa en comparación con otros tratamientos no específicos (como la relajación estándar) siguen haciendo necesarias más investigaciones. A pesar de estas limitaciones, es interesante comentar brevemente sus principales resultados. Estos, basados en los estudios de Shapiro et al. (30, 35, 37), Rosenzweig et al. (38), Jain et al. (39), Vieten y Astin (40) y Klatt et al. (41), fueron que el programa tuvo un efecto positivo inespecífico significativo en comparación con la ausencia de cualquier tratamiento (lista de espera). Pero en el único estudio (39) en el que se pudo comparar el programa con controles con tratamiento activo no hubo diferencias significativas entre ambos. Posteriormente concluyeron los mismos autores, con iguales limitaciones por la irregular metodología de los estudios, que el MBSR ha demostrado eficacia para muchas condiciones psiquiátricas y físicas y también en sujetos sanos (42).

El programa se ha mostrado efectivo en reducir el estrés, ansiedad y depresión (5) y se ha usado exitosamente en distintos trastornos de ansiedad (43-44) y 
ORIGINALES Y REVISIONES

en el estrés general $(36,45-46)$ en tanto que es capaz de reducir sus grados en las personas sanas. Se ha constatado su efectividad específicamente en el estrés de los profesores españoles de educación secundaria (47). Es beneficioso en la fobia social (48), el trastorno de ansiedad generalizada y el trastorno por angustia, en particular, observándose una mejoría significativa (49-51). El tratamiento cognitivo-conductual parece ser el tratamiento de elección en la fobia social, pero se ha sugerido (52) que el MBSR puede tener también algún beneficio en esos pacientes y que puede reducir en ellos la reactividad emocional mejorando la regulación de la misma $(48,53)$. El MBSR parece tener, en efecto, un impacto positivo clínicamente relevante en los procesos de regulación emocional (54). También se ha evidenciado (55) que la práctica de la meditación se asocia con disminuciones de las emociones negativas y la gravedad de los síntomas de ansiedad social, y que el MBSR supera a la intervención basada solo en el ejercicio físico aeróbico $(13,55)$. La bondad de las técnicas meditativas en los trastornos de ansiedad fue analizada en la revisión Cochrane de Krisanaprakornkit et al. (56) con resultados inconcluyentes. El pequeño número de estudios incluidos en la revisión no permitía extraer conclusiones sobre la eficacia de los programas de meditación en los trastornos de ansiedad. Las tasas de abandono durante las investigaciones fueron altas y no se informaron efectos adversos con la meditación. Algunos estudios sugieren que el MBSR puede ayudar a reducir ciertos factores (como la preocupación) que contribuyen a los problemas del sueño, según el estudio de revisión sistemática de Winbush et al. (57). Sin embargo, en su análisis de siete trabajos sobre el MBSR y trastornos del sueño, los autores no hallaron pruebas suficientes de que el programa mejorara significativamente la calidad del sueño y su duración. Más recientemente se ha propuesto el MBSR en un ensayo clínico piloto como una intervención útil en el insomnio crónico (58). Para la ansiedad en personas afectas de enfermedades crónicas, Bohlmeijer et al. (59) hallaron un moderado tamaño del efecto del MBSR de 0,47 , con considerable heterogeneidad.

\section{Depresión recurrente, resistente y residual}

El MBSR puede ser de utilidad en la depresión y la depresión resistente al tratamiento (12,60-61), en los trastornos del estado de ánimo y el estrés perinatales maternos (49), y hay indicios de que podría ser un recurso interesante para la ideación suicida (62). El programa disminuye asimismo los pensamientos rumiadores en los trastornos del estado de ánimo (63) y en poblaciones no clínicas (64). Los efectos de la introducción del programa al inicio de la psicoterapia, así como su efecto sobre el logro de las metas autodirigidas en contextos no psicoterapéuticos es superior a la psicoterapia sola (65). Bohlmeijer et al. (59) hallaron en personas afectas de enfermedades crónicas un pequeño tamaño del efecto medio global de 0,26 (IC del 95\%: 0,18-0,34) en seis estudios sobre la depresión, con ausencia de heterogeneidad. En el metaanálisis de Klainin-Yobas et al. (66) se ha constatado 
que las intervenciones basadas en conciencia plena son eficaces para aliviar síntomas depresivos en adultos con trastornos mentales y que dichas intervenciones podrían ser usadas de forma concurrente con otros tratamientos. Por último, parece que el MBSR puede ayudar a mejorar la calidad de vida relacionada con la salud y reducir los síntomas de depresión en los sujetos de residencias de ancianos (67).

\section{Cáncer}

Con relación al cáncer el MBSR es el programa de intervención más estudiado, a pesar de lo cual se necesitan más investigaciones para poder sacar conclusiones sobre su ayuda potencial. Hay hasta la fecha al respecto un metaanálisis (68), cinco revisiones sistemáticas (69-73) y una revisión narrativa (74), además de numerosos ensayos clínicos controlados. Speca et al. (75) comprobaron que mejoraba las alteraciones anímicas y los síntomas de estrés en una población heterogénea de enfermos de cáncer. Carlson et al. (76-77) usaron relajación, yoga suave y práctica diaria de meditación en casa en una investigación preliminar y concluyeron acerca de los beneficios del MBSR mediante la regulación de las concentraciones plasmáticas hormonales del eje hipotálamo-hipófiso-suprarrenal en pacientes con cáncer. Shapiro et al. (78) sugirieron que el MBSR parecía ser una intervención prometedora para mejorar la calidad del sueño en mujeres con cáncer de mama cuyas quejas de sueño eran debidas al estrés. Carlson y Garland (79) hallaron beneficios sobre la calidad del sueño en un grupo heterogéneo de pacientes con cáncer, corroborado con posterioridad (80). Por otra parte, Carlson et al. (81) demostraron también los beneficios del programa en la reducción del estrés y el aumento de la calidad de vida en los pacientes con cáncer de mama o próstata. Con relación al cáncer recurrente de próstata ya se había sugerido los beneficios del MBSR asociado a una dieta adecuada (82). El MBSR es viable para las mujeres recientemente diagnosticadas de cáncer de mama en estadio precoz y los resultados han proporcionado ciertas pruebas, asimismo preliminares, de sus efectos beneficiosos sobre la función inmune, calidad de vida, mejora en el estilo de afrontamiento de las pacientes (83) y en la mejora de su salud mental (84-85).

Otros estudios siguen sugiriendo que es una intervención beneficiosa en las pacientes con cáncer de mama, mejorando su ajuste psicosocial más allá de los efectos del tratamiento habitual y de una condición de control creíble (programa de educación nutricional) (86-87). Se ha propuesto, en fin, como ayuda para mejorar la calidad de vida en mujeres supervivientes a cáncer de mama (88-89) y como intervención de elección para tratar las secuelas psíquicas relacionadas con el cáncer (80). El programa puede mejorar en los pacientes con cáncer el ajuste psicosocial a su enfermedad (68), el estado psicológico y en particular el estado de ánimo $(73,90)$, algunos de los síntomas (como la fatiga), la inmunidad (91) y la calidad de vida $(81,92-95)$. Los resultados beneficiosos sobre la ansiedad y depresión relacionadas con supervivientes al cáncer de mama se han mantenido, con tamaño 
del efecto medio-grande, a los 12 meses de seguimiento en el ensayo controlado de Würtzen et al. (90). Sin embargo, para la mayoría de los síntomas físicos es improbable una mejoría significativa con el MBSR (73). Hay evidencias preliminares de que el MBSR en modo audio-individual para pacientes con cáncer y quimioterapia puede beneficiar el estado de ánimo y la calidad de vida (96). Por último, Matousek et al. (97) hallaron mejoras significativas de los grados de tensión autorrelatados, sintomatología depresiva y otros síntomas médicos en mujeres afectas de cáncer de mama tras el programa MBSR. Dicha mejoría era concordante con los resultados de la "respuesta de cortisol al despertar" (CAR, del inglés cortisol awakening response), que se ha propuesto como marcador biológico fiable del funcionamiento del eje hipotálamo-hipófiso-suprarrenal.

\section{Otras enfermedades y ámbitos clínicos}

La revisión de Bishop (98) basada en los estudios controlados sobre población general de Astin (99) y Shapiro et al. (30), y los estudios sobre población clínica de Speca et al. (75) y Teasdale et al. (100), además de en otros estudios no controlados, constató que no había suficientes evidencias para recomendar encarecidamente el MBSR, pero reconocía que había mejora, en los pacientes con enfermedades crónicas, de la autogestión del estrés y de los síntomas del estado de ánimo asociados. En un conjunto de 83 pacientes con enfermedades crónicas, el aumento en la conciencia plena tras la práctica de MBSR estuvo relacionado significativamente con la reducción de los síntomas depresivos, estrés, síntomas médicos y con aumento en la sensación general de coherencia (101).

Davidson et al. (102) hallaron que los sujetos meditadores del programa MBSR tenían un incremento significativamente mayor de anticuerpos que los no meditadores del grupo control (lista de espera con un entrenamiento comparable), de modo que se concluyó que la meditación puede influir positivamente en la inmunidad. La mejora en el bienestar psíquico posterior al MBSR se asoció, en una muestra heterogénea de pacientes sin trastornos autoinmunes, con mayor actividad citolítica de las células natural killer y disminución de las concentraciones plasmáticas de proteína $C$ reactiva (103).

En un grupo reducido de epilépticos resistentes a los anticonvulsivantes la práctica de la meditación continuada durante un año fue de ayuda sustancial en la mejora de los trazados electroencefalográficos (104). En pacientes con psoriasis tratados con protocolo de luz ultravioleta, una meditación guiada por cinta de audio, dentro del MBSR, aumentó la tasa de resolución de las lesiones psoriásicas (105). En la interconsulta hospitalaria psiquiátrica en cardiología, la intervención grupal basada en la conciencia plena puede proporcionar a los pacientes medios adicionales de manejo de su angustia al tiempo que les ofrece contacto social y apoyo (106). La meditación por sí sola puede ser eficaz en los pacientes afectos de insuficiencia cardíaca crónica reduciendo la activación simpática, evaluada por 
la concentración plasmática de norepinefrina (107). En particular el MBSR podría tener un papel importante en los pacientes afectos de esa patología, según los resultados del estudio acerca del apoyo, educación e investigación en la insuficiencia cardíaca crónica (SEARCH) (108). El programa MBSR ha demostrado eficacia en la reducción de síntomas de ansiedad y depresión, estrés percibido, presión arterial e índice de masa corporal en pacientes con cardiopatía coronaria (109).

Bohlmeijer et al. (59) concluyen en su revisión metaanalítica basada en ocho estudios controlados y aleatorizados que en personas afectas de enfermedades crónicas el MBSR tiene un tamaño del efecto pequeño de 0,32 de mejora para el estrés psíquico (IC del 95\%: 0,13-0,50), con ausencia de heterogeneidad. El denominado "síndrome de angustia corporal" (bodily distress syndrome) (110) incluye la mayoría de pacientes con trastornos somatomorfos, y el MBSR se ha propuesto como un programa de prevención específica para ese grupo de pacientes de alto riesgo. El MBSR ya se había sugerido como una intervención útil en mujeres afectas de síndrome de intestino irritable (111).

En relación con el virus de la inmunodeficiencia humana (Vih) el programa MBSR podría tener un efecto positivo en los ámbitos relacionados con la hostilidad, relaciones interpersonales, rendimiento escolar y salud física en jóvenes urbanos infectados (112). Hay también algunas pruebas preliminares de que el entrenamiento en meditación puede amortiguar la disminución de linfocitos T cd4 en pacientes adultos con el tipo Vih1 (113), puede mejorar la actividad de las células natural killer en pacientes con Vih (114) y mejorar los síntomas médicos autorreferidos (115). En el ensayo clínico de Duncan et al. (116) los pacientes con Vih que siguieron el programa experimentaron a los tres y seis meses de finalizado una reducción de la frecuencia de efectos secundarios atribuibles a los tratamientos antirretrovirales y mejoraron la angustia relacionada con los síntomas de la enfermedad. En varones homosexuales con Vih el programa ha mostrado también que los incrementos en conciencia plena correlacionaban significativamente a los seis meses con disminución en la evitación experiencial, el afecto positivo y con mejoría de los síntomas depresivos (117).

El programa parece mejorar la calidad de vida y el estrés en los pacientes con artritis reumatoide (118), asma persistente (119), diabetes tipo II (120-121) e hipertensión arterial (122), entre otras enfermedades, y parece disminuir la respuesta inflamatoria provocada por estrés (123). También puede reducir la expresión proinflamatoria de genes en adultos mayores (124). Puede ser asimismo un recurso eficaz para disminuir las molestias cognitivas y no cognitivas relacionadas con los sofocos y sudores nocturnos en mujeres peri y posmenopáusicas (125). En pacientes con traumatismo craneoencefálico, el MBSR mejoró tanto la fatiga mental como el funcionamiento neuropsicológico (126). Pero estudios preliminares, a falta de ensayos controlados, no han mostrado efectividad del programa en obesos con conductas de ingesta impulsiva y dietas inadecuadas (127). 
ORIGINALES Y REVISIONES

Estudios recientes epidemiológicos han demostrado una asociación entre el estrés mental percibido y el ictus isquémico. Las intervenciones basadas en conciencia plena, en general, han mostrado efectos beneficiosos en los pacientes que han sufrido ictus transitorios, según la revisión sistemática de Lawrence et al. (128) basada en 160 pacientes de cuatro ensayos clínicos. En sujetos que habían recibido órganos trasplantados el MBSR redujo la ansiedad, depresión y sueño poco reparador, y mejoró su calidad de vida durante un año de seguimiento (129).

\section{Otros ámbitos no clínicos}

En la aplicación del programa a un grupo de personas, mayoritariamente profesionales de la asistencia primaria de salud de Palma de Mallorca, la reducción en el malestar psíquico conllevó una disminución de la rumiación mental y de la afectividad negativa. Los resultados confirman, según los autores, la utilidad del programa (130) y confirman también hallazgos de estudios previos del mismo equipo investigador (131-132). El programa reduce el burnout y la disforia, y aumenta la conciencia plena y la empatía en profesionales de la asistencia primaria, y también produce cambios en esos profesionales hacia un mejor autocuidado y un mayor profesionalismo (133). Shapiro et al. (35) habían observado una disminución significativa en las cavilaciones respecto del valor basal en el grupo de MBSR, mientras que no se observaron diferencias en el grupo control. Estos resultados se confirmaron en el estudio realizado por Jain et al. (39). Por otra parte, el programa mejora el umbral de percepción consciente y la capacidad de memoria de trabajo visual (134), y mejora asimismo la disminución del pensamiento reflexivo en comparación con el entrenamiento de relajación (39). El programa aumentó significativamente la empatía en estudiantes de enfermería (29). En esta población parece, como ya se ha comentado, una intervención eficaz para disminuir el estrés y la ansiedad (34).

En el metaanálisis de Chiesa y Serretti (36), agregando los resultados pre y postratamiento el MBSR fue significativamente más eficaz en la mejora de la espiritualidad que los controles sin tratamiento activo. Usando el cuestionario «Indice de experiencias espirituales principales» (Inspirit) hubo una mejora significativa en los valores de espiritualidad en el grupo que recibió el programa de tratamiento -según los estudios de Astin (99) y Shapiro et al. (30)- frente a los controles inactivos. Cuando el comparador del programa fue otro tratamiento activo, el MBSR estaba correlacionado con un aumento significativo en los valores de espiritualidad que eran comparables, aunque no superiores, al tratamiento de comparación activo adicional (39). El estudio posterior de Geary y Rosenthal (135) ha mostrado que el MBSR reduce la tensión percibida y aumenta experiencias espirituales diarias, si bien su muestra era reducida y el grupo control no era activo. 
ORIGINALES Y REVISIONES

\section{Discusión y conclusiones}

A pesar de la abundancia de ensayos clínicos controlados su calidad es inconstante, de modo que los resultados no pueden ser conclusivos por más que haya buenas revisiones sistemáticas y metaanálisis. La mayoría de los ensayos analizados son de tamaño muestral reducido y de diseño clásico o en paralelo (el grupo control recibe otra intervención similar a la vez que el grupo experimental), pero se echan en falta estudios con diseño de no-inferioridad. En general, los resultados de nuestra revisión avalan la hipótesis subyacente al MBSR de que el programa enseña a observar situaciones y pensamientos sin hacer juicios ni reaccionar ante ellos de manera impulsiva, ayuda a las personas a desarrollar la atención selectiva (134), una conciencia más reflexiva de las experiencias internas y externas, y por eso parece ser un instrumento eficaz para la reducción del estrés global. En efecto, el estudio de Hölzel et al. (136) parece confirmar que el programa MBSR aumenta la concentración de materia gris en el hipocampo izquierdo, que se ha postulado juega un papel central en la mediación de algunos de los beneficios de la meditación debido a su implicación en la modulación de la excitación cortical y la capacidad de respuesta.

El MBSR puede ser de utilidad en un gran número de personas, tanto si sufren problemas clínicos como si no (8). Pero principalmente en la actualidad, igual que sucede con el tratamiento cognitivo basado en la conciencia plena (mindfulness based cognitive therapy o MBCT) y buscando aplicaciones terapéuticas cada vez más específicas el programa se utiliza, además de en enfermedades crónicas diversas, en pacientes que sufren episodios depresivos recurrentes, y en los que presentan síntomas depresivos residuales y son resistentes a otros tratamientos. En relación con las depresiones, el MBSR partió desde enfoques inespecíficos a otros más específicos incluyendo las depresiones, pero el MBCT nació específicamente para tratar las depresiones recurrentes. De hecho, este último y no el MBSR es el tratamiento basado en conciencia plena o mindfulness para las depresiones que más claramente está avalado por estudios empíricos de calidad suficiente.

Los pasos que se dan en la aplicación de la conciencia plena con el dolor crónico son, en primer lugar, ver y conocer qué siente el sujeto tanto corporal como emocionalmente en relación con ese dolor. En segundo lugar, se pretende reconocer las reacciones emocionales al mismo. Por último, se intenta aceptar el dolor momento a momento, sin pretender alejarnos de él. Además de mejorar el manejo del dolor y del estrés en condiciones médicas muy diversas, las pruebas sugieren que el MBSR es eficaz o efectivo como intervención adyuvante en los síntomas de ansiedad y salud mental en general, y en ciertos trastornos mentales, y que contribuye a mejorar la salud en general en poblaciones no clínicas. La relación entre los grados de estrés percibido y la espiritualidad sigue siendo incierta. 
Como conclusiones se puede decir que el programa MBSR es una intervención instructiva o psicoeducativa, estructurada, multimodal, diseñada para mejorar la conciencia plena de los participantes con focalización en el presente. El programa tiene muchos componentes activos, además de la meditación vipassana. Es, por tanto, una intervención inespecífica en la que no es posible discriminar cuál es su componente más activo. Hay pruebas de su efectividad en el dolor crónico (incluyendo la fibromialgia), depresiones recurrentes o con síntomas residuales, el estrés en general y en diversos trastornos de ansiedad, así como en ciertas condiciones médicas diversas como el cáncer, epilepsia resistente, psoriasis y enfermos con Vih, entre otras. En particular, con relación al dolor hay pruebas de que aceptarlo correlaciona con mejores resultados funcionales y emocionales que la reducción o distracción de la atención y de la vigilancia frente al mismo. Para terminar, el cuerpo probatorio que se ha acumulado hasta la fecha es muy prometedor y fuertemente indiciario de los beneficios del MBSR en el corto y medio plazo, pero se necesitan más estudios adecuados para confirmar su bondad por tiempos prolongados, y estudios potentes de buena calidad metodológica que confirmen su eficacia o efectividad.

\section{Bibliografia}

(1) Kabat-Zinn J. Full catastrophe living: using the wisdom of your body and mind to face stress, pain and illness. Nueva York (EEUU): Delacorte Press; 1990. [Traducción: Vivir con plenitud las crisis. Cómo utilizar la sabiduría del cuerpo y de la mente para afrontar el estrés, el dolor y la enfermedad. $4^{\text {a }}$ ed. Barcelona: Kairós; 2010.]

(2) Carmody J, Baer RA. How long does a mindfulness-based stress reduction program need to be? A review of class contact hours and effect sizes for psychological distress. J Clin Psychol. 2009;65:62738.

(3) Niazi AK, Niazi SK. Mindfulness-based stress reduction: a non-pharmacological approach for chronic illnesses. N Am J Med Sci. 2011;3:20-3. [**]

(4) Simpson J, Mapel T. An investigation into the health benefits of mindfulness-based stress reduction (MBSR) for people living with a range of chronic physical illnesses in New Zealand. N Z Med J. 2011;124:68-75. [*]

(5) Fjorback LO, Arendt M, Ornbøl E, Fink P, Walach H. Mindfulness-based stress reduction and mindfulness-based cognitive therapy: a systematic review of randomized controlled trials. Acta Psychiatr Scand. 2011;124:102-19. [**]

(6) Biegel GM, Brown KW, Shapiro SL, Schubert CM. Mindfulness-based stress reduction for the treatment of adolescent psychiatric outpatients: a randomized clinical trial. J Consult Clin Psychol. 2009;77:855-66. [*]

(7) Baer RA. Mindfulness training as a clinical intervention: a conceptual and empirical review. Clin Psychol Sci Prac. 2003;10:125-43. 
(8) Grossman P, Niemann L, Schmidt S, Walach H. Mindfulness-based stress reduction and health benefits. A meta-analysis. J Psychosom Res. 2004;57:35-43. [***]

(9) Hoffman BM, Papas RK, ChatkoffDK, Kerns RD. Meta-analysis of psychological interventions for chronic low back pain. Health Psychol. 2007;26:1-9.

(10) Kabat-Zinn J. An outpatient program in behavioral medicine for chronic pain patients based on the practice of mindfulness meditation: theoretical considerations and preliminary results. Gen Hosp Psychiatry. 1982;4:33-47.

(11) Kabat-Zinn J, Lipworth L, Burney R, Sellers W. Four year follow-up of a meditationbased program for the self-regulation of chronic pain: treatment outcomes and compliance. Clin J Pain. 1986;2:159-73.

(12) Kingston J, Chadwick P, Meron D, Skinner TC. A pilot randomized control trial investigating the effect of mindfulness practice on pain tolerance, psychological well-being, and physiological activity. J Psychosom Res. 2007;62:297-300.

(13) Merkes M. Mindfulness-based stress reduction for people with chronic diseases. Aust J Prim Health. 2010;16:200-10.

(14) Rosenzweig S, Greeson JM, Reibel DK, Green JS, Jasser SA, Beasley D. Mindfulness-based stress reduction for chronic pain conditions: variation in treatment outcomes and role of home meditation practice. J Psychosom Res. 2010;68:29-36.

(15) MacCoon DG, Imel ZE, Rosenkranz MA, Sheftel JG, Weng HY, Sullivan JC, et al. The validation of an active control intervention for Mindfulness Based Stress Reduction (MBSR). Behav Res Ther. 2012;50:3-12. [*]

(16) Kaplan KH, Goldenberg DL, Galvin-Nadeau M. The impact of a meditation-based stress reduction program on fibromyalgia. Gen Hosp Psychiatry. 1993;15:284-9.

(17) Grossman P, Tiefenthaler-Gilmer U, Raysz A, Kesper U. Mindfulness training as an intervention for fibromyalgia: evidence of postintervention and 3-year follow-up benefits in well-being. Psychother Psychosom. 2007;76:226-33.

(18) Smith BW, Shelley BM, Dalen J, Wiggins K, Tooley E, Bernard J. A pilot study comparing the effects of mindfulness-based and cognitive-behavioral stress reduction. J Altern Complement Med. 2008; $14: 251-8$.

(19) Lush E, Salmon P, Floyd A, Studts JL, Weissbecker I, Sephton SE. Mindfulness meditation for symptom reduction in fibromyalgia: psychophysiological correlates. J Clin Psychol Med Settings. 2009; 16:200-7.

(20) Sephton SE, Salmon P, Weissbecker I, Ulmer C, Floyd A, Hoover K, et al. Mindfulness meditation alleviates depressive symptoms in women with fibromyalgia: results of a randomized clinical trial. Arthritis Rheum. 2007;57:77-85. [*]

(21) Schmidt S, Grossman P, Schwarzer B, Jena S, Naumann J, Walach H. Treating fibromyalgia with mindfulness-based stress reduction: results from a 3-armed randomized controlled trial. Pain. 2011;152:361-9. [*]

(22) Morone NE, Greco CM, Rollman BL, Moore CG, Lane B, Morrow L, et al. The design and methods of the aging successfully with pain study. Contemp Clin Trials. 2012;33:417-25. [*]

(23) Wong SY, Chan FW, Wong RL, Chu MC, Kitty Lam YY, Mercer SW, et al. Comparing the effectiveness of mindfulness-based stress reduction and multidisciplinary intervention programs for chronic pain: a randomized comparative trial. Clin J Pain. 2011;27:724-34. [*] 
ORIGINALES Y REVISIONES

(24) Cramer H, Haller H, Lauche R, Dobos G. Mindfulness-based stress reduction for low back pain. A systematic review. BMC Complement Altern Med. 2012;12:162. [**]

(25) Veehof MM, Oskam MJ, Schreurs KM, Bohlmeijer ET. Acceptance-based interventions for the treatment of chronic pain: a systematic review and meta-analysis. Pain. 2011;152:533-42. [***]

(26) Plews-Ogan M, Owens JE, Goodman M, Wolfe P, Schorling J. A pilot study evaluating mindfulness-based stress reduction and massage for the management of chronic pain. J Gen Intern Med. 2005;20:1136-8. [*]

(27) Esmer G, Blum J, Rulf J, Pier J. Mindfulness-based stress reduction for failed back surgery syndrome: a randomized controlled trial. J Am Osteopath Assoc. 2010;110:646-52. Fe de erratas en: J Am Osteopath Assoc. 2011;111:3 y J Am Osteopath Assoc. 2011;111:424. [*]

(28) Shapiro SL, Brown KW, Thoresen C, Plante TG. The moderation of Mindfulness-based stress reduction effects by trait mindfulness: results from a randomized controlled trial. J Clin Psychol. 2011;67:267-77. [*]

(29) Davis M. A multidimensional approach to individual differences in empathy. JSAS Catalog of Selected Documents in Psychology. 1980;10:85.

(30) Shapiro SL, Schwartz GE, Bonner G. Effects of mindfulness-based stress reduction on mental and premedical students. J Behav Med. 1998;21:581-99.

(31) Shapiro SL, Oman D, Thoresen CE, Plante TG, Flinders T. Cultivating mindfulness: effects on well-being. J Clin Psychol. 2008;64:840-62. [*]

(32) Oman D, Shapiro SL, Thoresen CE, Plante TG, Flinders T. Meditation lowers stress and supports forgiveness among college students: a randomized controlled trial. J Am Coll Health. 2008;56:56978. [*]

(33) Cohen-Katz J, Wiley S, Capuano T, Baker DM, Deitrick L, Shapiro S. The effects of mindfulness-based stress reduction on nurse stress and burnout: a qualitative and quantitative study, part III. Holist Nurs Pract. 2005;19:78-86. [*]

(34) Kang YS, Choi SY, Ryu E. The effectiveness of a stress coping program based on mindfulness meditation on the stress, anxiety, and depression experienced by nursing students in Korea. Nurse Educ Today. 2009;29:538-43.

(35) Shapiro SL, Brown KW, Biegel GM. Teaching self-care to caregivers: effects of mindfulness-based. stress reduction on the mental health of therapists in training. Train Educ Prof Psychol. 2007;1:105-15.

(36) Chiesa A, Serretti A. Mindfulness-based stress reduction for stress management in healthy people: a review and meta-analysis. J Altern Complement Med. 2009;15:593-600. [***]

(37) Shapiro SL, Astin JA, Bishop SR, Cordova M, Mindfulness-based stress reduction for health. Care professionals: results from a randomized trial. Int J Stress Manag. 2005;12:164-76. [*]

(38) Rosenzweig S, Reibel DK, Greeson JM, Brainard GC, Hojat M. Mindfulness-based stress reduction lowers psychological distress in medical students. Teach Learn Med. 2003;15:88-92.

(39) Jain S, Shapiro SL, Swanick S, Roesch SC, Mills PJ, Bell I, et al. A randomized controlled trial of mindfulness meditation versus relaxation training: effects on distress, positive states of mind, rumination, and distraction. Ann Behav Med. 2007;33:11-21. [*]

(40) Vieten C, Astin J. Effects of a mindfulness-based intervention during pregnancy on prenatal stress and mood: results of a pilot study. Arch Womens Ment Health. 2008;11:67-74.

(41) Klatt MD, Buckworth J, Malarkey WB. Effects of low-dose mindfulness-based stress reduction (MBSR-ld) on working adults. Health Educ Behav. 2009;36:601-14. [*] 
(42) Chiesa A, Serretti A. A systematic review of neurobiological and clinical features of mindfulness meditations. Psychol Med. 2010;40:1239-52. [**]

(43) Vøllestad J, Sivertsen B, Nielsen GH. Mindfulness-based stress reduction for patients with anxiety disorders: evaluation in a randomized controlled trial. Behav Res Ther. 2011;49:281-8. [*]

(44) Arch JJ, Ayers CR, Baker A, Almklov E, Dean DJ, Craske MG. Randomized clinical trial of adapted mindfulness-based stress reduction versus group cognitive behavioral therapy for heterogeneous anxiety disorders. Behav Res Ther. 2013;51:185-96. [*]

(45) Nyklícek I, Kuijpers KF. Effects of mindfulness-based stress reduction intervention on psychological well-being and quality of life: is increased mindfulness indeed the mechanism? Ann Behav Med. 2008;35:331-40. [*]

(46) Praissman S. Mindfulness-based stress reduction: a literature review and clinician's guide. J Am Acad Nurse Pract. 2008;20:212-6. [**]

(47) Franco C, Mañas I, Cangas AJ, Moreno E, Gallego J. Reducing teachers' psychological distress through a mindfulness training program. Spanish J Psychol. 2010;13:655-66.

(48) Goldin P, Ramel W, Gross J. Mindfulness meditation training and self-referential processing in social anxiety disorder: behavioral and neural effects. J Cog Psychother. 2009;23:242-57.

(49) Kabat-Zinn J, Massion AO, Kristeller J, Peterson LG, Fletcher KE, Pbert L, Lenderking WR, Santorelli SF. Effectivenes of a meditation-based stress reduction program in the treatment of anxiety disorders. Am J Psychiatry. 1992;149:936-43.

(50) Miller JJ, Fletcher K, Kabat-Zinn J. Three-year follow-up and clinical implications of a mindfulness meditation-based stress reduction intervention in the treatment of anxiety disorders. Gen Hosp Psychiatry. 1995;17:192-200.

(51) Lee SH, Ahn SC, Lee YJ, Choi TK, Yook KH, Suh SY. Effectiveness of a meditation-based stress management program as an adjunct to pharmacotherapy in patients with anxiety disorder. J Psychosom Res. 2007;62:189-95.

(52) Koszycki D, Benger M, Shlik J, Bradwejn J. Randomized trial of a meditation-based stress reduction program and cognitive behavior therapy in generalized social anxiety disorder. Behav Res Ther. 2007;45:2518-26. [*]

(53) Goldin PR, Gross JJ. Effects of mindfulness-based stress reduction (MBSR) on emotion regulation in social anxiety disorder. Emotion. 2010;10:83-91. [+]

(54) Robins CJ, Keng SL, Ekblad AG, Brantley JG. Effects of mindfulness-based stress reduction on emotional experience and expression: a randomized controlled trial. J Clin Psychol. 2012;68:117-31. [*]

(55) Goldin P, Ziv M, Jazaieri H, Hahn K, Gross JJ. MBSR vs aerobic exercise in social anxiety: fMRI of emotion regulation of negative self-beliefs. Soc Cog Affect Neurosci. 2013;8:65-72.

(56) Krisanaprakornkit T, Krisanaprakornkit W, Piyavhatkul N, Laopaiboon M. Meditation therapy for anxiety disorders. Cochrane Database Syst Rev. 2006;(1):CD004998. [**]

(57) Winbush NY, Gross CR, Kreitzer MJ. The effects of mindfulness-based stress reduction on sleep disturbance: a systematic review. Explore (NY). 2007;3:585-91. [**]

(58) Gross CR, Kreitzer MJ, Reilly-Spong M, Wall M, Winbush NY, Patterson R, et al. Mindfulness-based stress reduction versus pharmacotherapy for chronic primary insomnia: a randomized controlled clinical trial. Explore (NY). 2011;7:76-87. [*]

(59) Bohlmeijer E, Prenger R, Taal E, Cuijpers P. The effects of mindfulness-based stress reduction therapy on mental health of adults with a chronic medical disease: a meta-analysis. J Psychosom Res. 2010;68:539-44. [***] 
(60) Kenny MA, Williams JM. Treatment-resistant depressed patients show a good response to mindfulness-based cognitive therapy. Behav Res Ther. 2007;45:617-25.

(61) Harley R, Sprich S, Safren S, Jacobo M, Fava M. Adaptation of dialectical behavior therapy skills training group for treatment-resistant depression. J Nerv Ment Dis. 2008;196:136-43.

(62) Birnbaum L, Birnbaum A. In search of inner wisdom: guided mindfulness meditation in the context of suicide. ScientificWorldJournal. 2004;4:216-27.

(63) Ramel W, Goldin PR, Carmona PE, McQuaid JR. The effects of mindfulness meditation on cognitive processes and affect in patients with past depression. Cognit Ther Res. 2004;28:433-55.

(64) Deyo M, Wilson KA, Ong J, Koopman C. Mindfulness and rumination: does mindfulness training lead to reductions in the ruminative thinking associated with depression? Explore (NY). 2009;5:265-71. [+]

(65) Weiss M, Nordlie JW, Siegel EP. Mindfulness-based stress reduction as an adjunct to outpatient psychotherapy. Psychother Psychosom. 2005;74:108-12. [+]

(66) Klainin-Yobas P, Cho MA, Creedy D. Efficacy of mindfulness-based interventions on depressive symptoms among people with mental disorders: a meta-analysis. Int J Nurs Stud. 2012;49:109-21. [***]

(67) Ernst S, Welke J, Heintze C, Gabriel R, Zöllner A, Kiehne S, et al. Effects of mindfulness-based stress reduction on quality of life in nursing home residents: a feasibility study. Forsch Komplementmed. 2008;15:74-81. [*]

(68) Ledesma D, Kumano H. Mindfulness-based stress reduction and cancer: a meta-analysis. Psychooncology. 2009;18:571-9. [***]

(69) Ott MJ, Norris RL, Bauer-Wu SM. What is the evidence for the use of mindfulness-based interventions in cancer care? A review. Integr Cancer Ther. 2006;5:98-108.

(70) Smith JE, Richardson J, Hoffman C, Pilkington K. Mindfulness-Based Stress Reduction as supportive therapy in cancer care: systematic review. J Adv Nurs. 2005;52:315-27. [**]

(71) Matchim Y, Armer JM. Measuring the psychological impact of mindfulness meditation on health among patients with cancer: a literature review. Oncol Nurs Forum. 2007;34:1059-66. [**]

(72) Shennan C, Payne S, Fenlon D. What is the evidence for the use of mindfulness-based interventions in cancer care? A review. Psychooncology. 2011;20:681-97.

(73) Musial F, Büssing A, Heusser P, Choi KE, Ostermann T. Mindfulness-based stress reduction for integrative cancer care: a summary of evidence. Forsch Komplementmed. 2011;18:192-202. [**]

(74) Mackenzie MJ, Carlson LE, Speca M. Mindfulness-based stress reduction (MBSR) in oncology: rationale and review. Evid Base Integr Med 2005;2:139-45.

(75) Speca M, Carlson LE, Goodey E, Angen M. A randomized, wait-list controlled clinical trial: the effect of a mindfulness meditation-based stress reduction program on mood and symptoms of stress in cancer outpatients. Psychosom Med. 2000;62:613-22. [*]

(76) Carlson LE, Speca M, Patel KD, Goodey E. Mindfulness-based stress reduction in relation to quality of life, mood, symptoms of stress, and immune parameters in breast and prostate cancer outpatients. Psychosom Med. 2003;65:571-81.

(77) Carlson LE, Speca M, Patel KD, Goodey E. Mindfulness-based stress reduction in relation to quality of life, mood, symptoms of stress and levels of cortisol, dehydroepiandrosterone sulfate (DHEAS) and melatonin in breast and prostate cancer outpatients. Psychoneuroendocrinology. 2004;29:448-74. [+]

(78) Shapiro SL, Bootzin RR, Figueredo AJ, Lopez AM, Schwartz GE. The efficacy of mindfulness-based stress reduction in the treatment of sleep disturbance in women with breast cancer: an exploratory study. J Psychosom Res. 2003;54:85-91. [*] 
(79) Carlson LE, Garland SN. Impact of mindfulness-based stress reduction (MBSR) on sleep, mood, stress and fatigue symptoms in cancer outpatients. Int J Behav Med. 2005;12:278-85. [+]

(80) Garland SN, Carlson LE, Antle MC, Samuels C, Campbell T. I-CAN SLEEP: rationale and design of a non-inferiority RCT of mindfulness-based stress reduction and cognitive behavioral therapy for the treatment of insomnia in cancer survivors. Contemp Clin Trials. 2011;32:747-54. [*]

(81) Carlson LE, Speca M, Faris P, Patel KD. One year pre-post intervention follow-up of psychological, immune, endocrine and blood pressure outcomes of mindfulness-based stress reduction (MBSR) in breast and prostate cancer outpatients. Brain Behav Immun. 2007;21:1038-49. [+]

(82) Saxe GA, Hébert JR, Carmody JF, Kabat-Zinn J, Rosenzweig PH, Jarzobski D, et al. Can diet in conjunction with stress reduction affect the rate of increase in prostate specific antigen after biochemical recurrence of prostate cancer? J Urol. 2001;166:2202-7. [+]

(83) Witek-Janusek L, Albuquerque K, Chroniak KR, Chroniak C, Durazo-Arvizu R, Mathews HL. Effect of mindfulness based stress reduction on immune function, quality of life and coping in women newly diagnosed with early stage breast cancer. Brain Behav Immun. 2008;22:969-81. [+]

(84) Cramer H, Lauche R, Paul A, Dobos G. Mindfulness-based stress reduction for breast cancer-a systematic review and meta-analysis. Curr Oncol. 2012;19:e343-52. [***]

(85) Zainal NZ, Booth S, Huppert FA. The efficacy of mindfulness-based stress reduction on mental health of breast cancer patients: a meta-analysis. Psychooncology. 2013;22:1457-65. [***]

(86) Henderson VP, Clemow L, Massion AO, Hurley TG, Druker S, Hébert JR. The effects of mindfulness-based stress reduction on psychosocial outcomes and quality of life in early-stage breast cancer patients: a randomized trial. Breast Cancer Res Treat. 2012;131:99-109. [*]

(87) Henderson VP, Massion AO, Clemow L, Hurley TG, Druker S, Hébert JR. A randomized controlled trial of mindfulness-based stress reduction for women with early-stage breast cancer receiving radiotherapy. Integr Cancer Ther. 2013;12:404-13. [*]

(88) Lengacher CA, Johnson-Mallard V, Post-White J, Moscoso MS, Jacobsen PB, Klein TW, et al. Randomized controlled trial of mindfulness-based stress reduction (MBSR) for survivors of breast cancer. Psychooncology. 2009;18:1261-72. [*]

(89) Lerman R, Jarski R, Rea H, Gellish R, Vicini F. Improving symptoms and quality of life of female cancer survivors: a randomized controlled study. Ann Surg Oncol. 2012;19:373-8. [*]

(90) Würtzen H, Dalton SO, Elsass P, Sumbundu AD, Steding-Jensen M, Karlsen RV, et al. Mindfulness significantly reduces self-reported levels of anxiety and depression: results of a randomised controlled trial among 336 Danish women treated for stage I-III breast cancer. Eur J Cancer. 2013;49:1365-73. [*]

(91) Lengacher CA, Kip KE, Post-White J, Fitzgerald S, Newton C, Barta M, et al. Lymphocyte recovery after breast cancer treatment and mindfulness-based stress reduction (MBSR) therapy. Biol Res Nurs. 2013;15:37-47. [*]

(92) Lengacher CA, Johnson-Mallard V, Barta M, Fitzgerald S, Moscoso MS, Post-White J, et al. Feasibility of a mindfulness-based stress reduction program for early-stage breast cancer survivors. $\mathrm{J}$ Holist Nurs. 2011;29:107-17.

(93) Lengacher CA, Reich RR, Post-White J, Moscoso M, Shelton MM, Barta M, et al. Mindfulness based stress reduction in post-treatment breast cancer patients: an examination of symptoms and symptom clusters. J Behav Med. 2012;35:86-94. [*]

(94) Hoffman CJ, Ersser SJ, Hopkinson JB, Nicholls PG, Harrington JE, Thomas PW. Effectiveness of mindfulness-based stress reduction in mood, breast- and endocrine-related quality of life, and well-being in stage 0 to III breast cancer: a randomized, controlled trial. J Clin Oncol. 2012;30:1335-42. [*] 
(95) Hoffman CJ, Ersser SJ, Hopkinson JB. Mindfulness-based stress reduction in breast cancer: a qualitative analysis. Complement Ther Clin Pract. 2012;18:221-6. [*]

(96) Altschuler A, Rosenbaum E, Gordon P, Canales S, Avins AL. Audio recordings of mindfulness-based stress reduction training to improve cancer patients' mood and quality of life--a pilot feasibility study. Support Care Cancer. 2012;20:1291-7. [**]

(97) Matousek RH, Pruessner JC, Dobkin PL. Changes in the cortisol awakening response (CAR) following participation in mindfulness-based stress reduction in women who completed treatment for breast cancer. Complement Ther Clin Pract. 2011;17:65-70. [+]

(98) Bishop SR. What do we really know about mindfulness-based stress reduction? Psychosom Med. 2002;64:71-83. Fe de erratas en: Psychosom Med. 2002;64:449. [**]

(99) Astin JA. Stress reduction through mindfulness meditation. Effects on psychological symptomatology, sense of control, and spiritual experiences. Psychother Psychosom. 1997;66:97-106.

(100) Teasdale JD, Segal ZV, Williams JM, Ridgeway VA, Soulsby JM, Lau MA. Prevention of relapse/recurrence in major depression by mindfulness-based cognitive therapy. J Consult Clin Psychol. 2000;68:615-23.

(101) Dobkin PL, Zhao Q. Increased mindfulness--the active component of the mindfulness-based stress reduction program? Complement Ther Clin Pract. 2011;17:22-7. [+]

(102) Davidson RJ, Kabat-Zinn J, Schumacher J, Rosenkranz M, Muller D, Santorelli SF, et al. Alterations in brain and immune function produced by mindfulness meditation. Psychosom Med. 2003;65:564-70.

(103) Fang CY, Reibel DK, Longacre ML, Rosenzweig S, Campbell DE, Douglas SD. Enhanced psychosocial well-being following participation in a mindfulness-based stress reduction program is associated with increased natural killer cell activity. J Altern Complement Med. 2010;16:531-8. [+]

(104) Deepak KK, Manchanda SK, Maheshwari MC. Meditation improves clinicoelectroencephalographic measures in drug-resistant epileptics. Biofeedback Self Regul. 1994;19:25-40.

(105) Kabat-Zinn J, Wheeler E, Light T, Skillings A, Scharf MJ, Cropley TG, et al. Influence of a mindfulness meditation-based stress reduction intervention on rates of skin clearing in patients with moderate to severe psoriasis undergoing phototherapy (UVB) and photochemotherapy (PUVA). Psychosom Med. 1998;60:625-32.

(106) Nickels MW, Privitera MR, Coletta M, Sullivan P. Treating depression: psychiatric consultation in cardiology. Cardiol J. 2009;16:279-93.

(107) Curiati JA, Bocchi E, Freire JO, Arantes AC, Braga M, Garcia Y, et al. Meditation reduces sympathetic activation and improves the quality of life in elderly patients with optimally treated heart failure: a prospective randomized study. J Altern Complement Med. 2005;11:465-72. [*]

(108) Sullivan MJ, Wood L, Terry J, Brantley J, Charles A, McGee V, et al. The Support, Education, and Research in Chronic Heart Failure Study (SEARCH): a mindfulness-based psychoeducational intervention improves depression and clinical symptoms in patients with chronic heart failure. Am Heart J. 2009;157:84-90.

(109) Parswani MJ, Sharma MP, Iyengar S. Mindfulness-based stress reduction program in coronary heart disease: A randomized control trial. Int J Yoga. 2013;6:111-7. [*]

(110) Fjorback LO. Mindfulness and bodily distress. Dan Med J. 2012;59:B4547. [**]

(111) Gaylord SA, Whitehead WE, Coble RS, Faurot KR, Palsson OS, Garland EL, et al. Mindfulness for irritable bowel syndrome: protocol development for a controlled clinical trial. BMC Complement Altern Med. 2009;9:24. [*] 
(112) Sibinga EM, Kerrigan D, Stewart M, Johnson K, Magyari T, Ellen JM. Mindfulness-based stress reduction for urban youth. J Altern Complement Med. 2011;17:213-8.

(113) Creswell JD, Myers HF, Cole SW, Irwin MR. Mindfulness meditation training effects on CD4+ T lymphocytes in HIV-1 infected adults: a small randomized controlled trial. Brain Behav Immun. 2009;23:184-8. [*]

(114) Robinson FP, Mathews HL, Witek-Janusek L. Psycho-endocrine-immune response to mindfulness-based stress reduction in individuals infected with the human immunodeficiency virus: a quasiexperimental study. J Altern Complement Med. 2003;9:683-94. [+]

(115) SeyedAlinaghi S, Jam S, Foroughi M, Imani A, Mohraz M, Djavid GE, et al. Randomized controlled trial of mindfulness-based stress reduction delivered to human immunodeficiency virus-positive patients in Iran: effects on CD4+ T lymphocyte count and medical and psychological symptoms. Psychosom Med. 2012;74:620-7. [*]

(116) Duncan LG, Moskowitz JT, Neilands TB, Dilworth SE, Hecht FM, Johnson MO. Mindfulness-based stress reduction for HIV treatment side effects: a randomized, wait-list controlled trial. J Pain Symptom Manage. 2012;43:161-71. [*]

(117) Gayner B, Esplen MJ, DeRoche P, Wong J, Bishop S, Kavanagh L, et al. A randomized controlled trial of mindfulness-based stress reduction to manage affective symptoms and improve quality of life in gay men living with HIV. J Behav Med. 2012;35:272-85. [*]

(118) Pradhan EK, Baumgarten M, Langenberg P, Handwerger B, Gilpin AK, Magyari T, et al. Effect of Mindfulness-Based Stress Reduction in rheumatoid arthritis patients. Arthritis Rheum. 2007;57:1134-42. [*]

(119) Pbert L, Madison JM, Druker S, Olendzki N, Magner R, Reed G, et al. Effect of mindfulness training on asthma quality of life and lung function: a randomised controlled trial. Thorax. 2012;67:76976. [*]

(120) Rosenzweig S, Reibel DK, Greeson JM, Edman JS, Jasser SA, McMearty KD, et al. Mindfulness-based stress reduction is associated with improved glycemic control in type 2 diabetes mellitus: a pilot study. Altern Ther Health Med. 2007;13:36-8. [+]

(121) Hartmann M, Kopf S, Kircher C, Faude-Lang V, Djuric Z, Augstein F, et al. Sustained effects of a mindfulness-based stress-reduction intervention in type 2 diabetic patients: design and first results of a randomized controlled trial (the Heidelberger Diabetes and Stress-study). Diabetes Care. 2012;35:945-7. [*]

(122) Palta P, Page G, Piferi RL, Gill JM, Hayat MJ, Connolly AB, et al. Evaluation of a mindfulness-based intervention program to decrease blood pressure in low-income African-American older adults. J Urban Health. 2012;89:308-16. [*]

(123) Rosenkranz MA, Davidson RJ, Maccoon DG, Sheridan JF, Kalin NH, Lutz A. A comparison of mindfulness-based stress reduction and an active control in modulation of neurogenic inflammation. Brain Behav Immun. 2013;27:174-84. [*]

(124) Creswell JD, Irwin MR, Burklund LJ, Lieberman MD, Arevalo JM, Ma J, et al. Mindfulness-Based Stress Reduction training reduces loneliness and pro-inflammatory gene expression in older adults: a small randomized controlled trial. Brain Behav Immun. 2012;26:1095-101. [*]

(125) Carmody JF, Crawford S, Salmoirago-Blotcher E, Leung K, Churchill L, Olendzki N. Mindfulness training for coping with hot flashes: results of a randomized trial. Menopause. 2011;18:611-20. [*]

(126) Johansson B, Bjuhr H, Rönnbäck L. Mindfulness-based stress reduction (MBSR) improves long-term mental fatigue after stroke or traumatic brain injury. Brain Inj. 2012;26:1621-8. [*] 
(127) Kearney DJ, Milton ML, Malte CA, McDermott KA, Martinez M, Simpson TL. Participation in mindfulness-based stress reduction is not associated with reductions in emotional eating or uncontrolled eating. Nutr Res. 2012;32:413-20.

(128) Lawrence M, Booth J, Mercer S, Crawford E. A systematic review of the benefits of mindfulness-based interventions following transient ischemic attack and stroke. Int J Stroke. 2013;8:465-74.

(129) Gross CR, Kreitzer MJ, Thomas W, Reilly-Spong M, Cramer-Bornemann M, Nyman JA, et al. Mindfulness-based stress reduction for solid organ transplant recipients: a randomized controlled trial. Altern Ther Health Med. 2010;16:30-8. [*]

(130) Martín Asuero A, García Banda G. The mindfulness-based stress reduction program (MBSR) reduces stress-related psychological distress in healthcare professionals. Span J Psychol. 2010;13:897-905.

(131) Martín Asuero A, García Banda G, Benito Oliver E. Reducción del estrés mediante atención plena: la técnica MBSR en la formación de profesionales de la salud. An Mod Conducta. 2005;31:557-71.

(132) Martín Asuero A, García Banda G. Las ventajas de estar presente: desarrollando una conciencia plena para reducir el malestar psicológico. Int J Clin Health Psychol. 2007;7:369-84.

(133) Martín Asuero A, Rodríguez Blanco T, Pujol Ribera E, Berenguera A, Moix Queraltó J. Evaluación de la efectividad de un programa de mindfulness en profesionales de atención primaria. Gac Sanit. 2013;27:521-8.

(134) Jensen CG, Vangkilde S, Frokjaer V, Hasselbalch SG. Mindfulness training affects attentionor is it attentional effort? J Exp Psychol Gen. 2012;141:106-23. [*]

(135) Geary C, Rosenthal SL. Sustained impact of MBSR on stress, well-being, and daily spiritual experiences for 1 year in academic health care employees. J Altern Complement Med. 2011;17:939-44. [+]

(136) Hölzel BK, Carmody J, Vangel M, Congleton C, Yerramsetti SM, Gard T, Lazar SW. Mindfulness practice leads to increases in regional brain gray matter density. Psychiatry Res. 2011;191:3643. $[+]$ 